Trauma Berufskrankh 2016 · [Suppl 4]:

18:S332-S339

DOI 10.1007/s10039-016-0145-6

Online publiziert: 25. April 2016

(c) Springer-Verlag Berlin Heidelberg 2016

CrossMark

M. Schnetzke - T. Guehring • P. A. Grützner

BG - Klinik Ludwigshafen, Ludwigshafen, Deutschland

\title{
Diagnostik und Therapie der akuten und chronischen Ellenbogeninstabilität
}

nern: dem Ulnohumeral-, dem Radiohumeral- und dem proximalen Radioulnargelenk.

Die wichtigsten Bandstrukturen am Ellenbogengelenk setzen sich aus dem medialen Kollateralband (MCL) sowie dem lateralen Kollateralbandkomplex (LCL-Komplex) zusammen. Das MCL stabilisiert bei Valgusstress und besteht aus drei Fasersträngen: dem anterioren, dem posterioren und dem transversalen Bündel (• Abb. 1).

Der LCL-Komplex stabilisiert bei Varusstress und setzt sich aus vier verschiedenen Strukturen zusammen: dem lateralen ulnaren Seitenband (LUCL), dem akzessorischen lateralen Seitenband, dem Lig. anulare radii sowie dem lateralen radialen Seitenband (•Abb. 2).

Die Stabilität des Ellenbogens beruht dabei auf dem Zusammenspiel von Gelenken, Unterarmmuskeln, knöchernen und ligamentären Strukturen, die nach

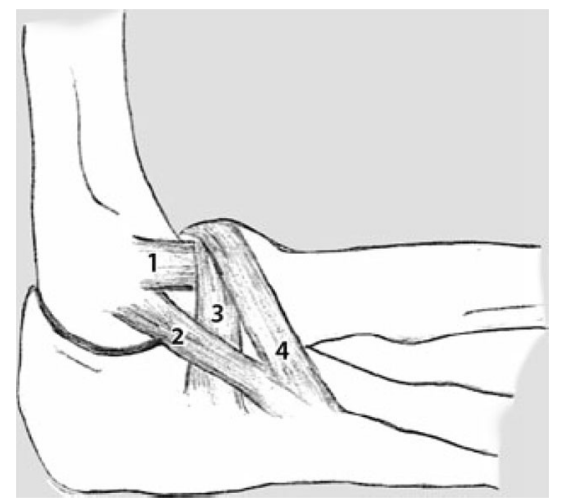

Abb. 2 ॥ Lateraler Kollateralbandkomplex (LCL-Komplex): 1 radiales Seitenband (RCL); 2 laterales ulnares Seitenband (LUCL); 3 Lig. anulare radii; 4 akzessorisches laterales Seitenband. (Aus [29])
O'Driscoll et al. [20] in primäre und sekundäre Stabilisatoren unterteilt werden (• Tab. 1).

\section{Ätiologie und Klassifikation}

Eine einfache Einteilung der Ellenbogeninstabilitäten existiert nicht. Instabilitäten am Ellenbogen können anhand verschiedener Kriterien eingeteilt werden (- Tab. 2).

\section{Ursache der Instabilität:}

traumatisch oder rezidivierende Mikrotraumata

Traumatische Luxationen des Ellenbogens stellen die häufigste Ursache für akute Instabilitäten des Ellenbogengelenkes dar. Mit einer Inzidenz von 6 bis 13/100.000 Luxationen pro Jahr stellt die traumatische Ellenbogenluxation nach der Schulterluxation die zweithäufigs-

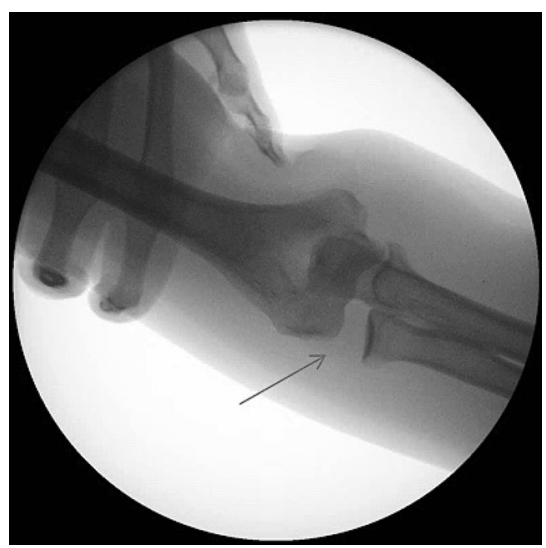

Abb. $3 \Delta$ Durchleuchtung des Ellenbogens nach Reposition mit ausgeprägter radialer Instabilität (Pfeil) im Varusstresstest 
Tab. 1 Übersicht über primäre und sekundäre Stabilisatoren des Ellenbogens

\begin{tabular}{|ll}
\hline Primäre Stabilisatoren & Ulnohumeralgelenk \\
\cline { 2 - 2 } & Mediales Kollateralband (MCL) \\
\cline { 2 - 2 } Sekundäre Stabilisatoren & Lateraler Kollateralbandkomplex (LCL-Komplex) \\
\cline { 2 - 2 } & Anteriore Gelenkkapsel \\
\hline Radiuskopf \\
\hline Extensoren und Flexoren des Unterarms
\end{tabular}

Tab. 2 Klassifikationen der Ellenbogeninstabilität. (Mod. nach [16])

\begin{tabular}{|ll}
\hline Kriterium & Formen der Instabilität \\
\hline Ursache & Traumatisch - rezidivierende Mikrotraumata \\
\hline Verletzungsmuster & Ligamentär - knöchern \\
\hline Zeitlicher Verlauf & Akut - rezidivierend - chronisch \\
\hline Grad der Instabilität & Subluxation - vollständige Luxation \\
\hline Richtung der Instabilität & Varus - valgus - posterolateral - posteromedial
\end{tabular}

te Luxation der großen Gelenke bei Erwachsenen und die häufigste in der Pädiatrie dar [11]. Als häufigster Verletzungsmechanismus wird dabei der Sturz auf den ausgetreckten und auBenrotierten Arm angesehen [18]. Nach O'Driscoll [17] ist die typische Luxation als Endstadium einer Weichteilruptur zu werten, die von lateral nach medial fortschreitet (sog. Ringtheorie). In der aktuellen Literatur finden sich jedoch immer mehr Erkenntnisse, die auch alternative Verletzungsmechanismen mit isolierter Ruptur des MCL postulieren, was im Gegensatz zu O'Driscolls Annahme steht [28].

Im Gegensatz zur traumatischen akuten Ellenbogeninstabilität stellen rezidivierende Mikrotraumata bei der Ausübung prädisponierender Berufe oder Sportarten (insbesondere Wurfsportarten) eine häufige Ursache für chronische Instabilitäten am Ellenbogen dar [25].

\section{Verletzungsmuster: ligamentäre oder knöcherne Instabilität}

In der englischsprachigen Literatur werden rein weichteilige Verletzungsmuster nach Luxation des Ellenbogens unter dem Begriff der „einfachen“ Luxation subsumiert [7]. Diese Terminologie ist irreführend, da es sich bei der ,einfachen" Luxation in der Regel um komplexe Weichteilverletzungen handelt [4]. Manche Autoren schließen knöcherne Bandausrisse des lateralen und medialen Epi- kondylus und Koronoidspitzenfrakturen vom Typ I nach Regan und Morrey in die Definition der einfachen Ellenbogenluxation mit ein [3, 27].

Die komplexe Ellenbogenluxation ist als eine Verletzung definiert, die den Ellenbogen durch eine Beschädigung der Gelenkfläche und der ligamentären Strukturen destabilisiert [14]. Die komplexe Ellenbogenluxation ist mit folgenden Frakturen vergesellschaftet:

- Koronoidfrakturen (Typ II und III nach Regan und Morrey),

- Radiuskopffrakturen,

- Transolekranonluxationsfrakturen.

Zeitlicher Verlauf: akut, rezidivierend oder chronisch

Nach dem zeitlichen Verlauf kann zwischen akuten, rezidivierenden und chronischen Instabilitäten am Ellenbogengelenk unterschieden werden [16]. Akute Instabilitäten entstehen traumatisch als Folge von Luxationen des Ellenbogens. Das Übersehen von Luxationstendenzen oder die Fehleinschätzung scheinbar stabiler Gelenke kann zu einer rezidivierenden oder chronischen Instabilität führen. Die Unterscheidung zwischen einer rezidivierenden und einer chronischen Instabilität ist im Einzelfall schwer zu treffen. Grundsätzlich handelt es sich bei der rezidivierenden Instabilität um eine akute, wiederholte (Sub-)Luxation des Ellenbogengelenkes und bei der chronischen Instabilität um einen posttraumati- schen Endzustand nach einem zurückliegenden Trauma oder infolge chronischer Überlastung.

Die häufigste Ausprägung der chronischen Ellenbogeninstabilität ist die sog. posterolaterale Rotationsinstabilität (PLRI) [19]. Diese entsteht meist infolge einer ligamentären Verletzung nach Ellenbogenluxation mit einer Ruptur des biomechanisch bedeutenden LUCL [9]. Alternative Ursachen für die Entstehung einer PLRI stellen wiederholte Kortisoninjektionen oder operative Behandlungen bei Epicondylitis radialis mit daraus resultierender Schwächung des LUCL dar [9]. Eine zeitliche Abgrenzung, ab wann von einer chronischen Instabilität gesprochen werden kann, existiert nach Literaturangaben nicht.

\section{Diagnostik und Therapie}

\section{Akute, ligamentäre Instabilität}

Die akute Ellenbogenluxation stellt aufgrund der meist augenscheinlichen Deformität des Ellenbogengelenks im Normalfall eine Blickdiagnose dar. Unter diesen Umständen sollten eine weitere klinische Untersuchung bezüglich Bewegungsausmaß, Stabilität und Druckschmerzhaftigkeit sowie unnötige Manipulationen unterlassen werden. Bei Verdacht auf eine Ellenbogenluxation sollte nach Erfassung des Nerven- und Gefäßstatus eine Röntgendiagnostik in 2 Ebenen durchgeführt werden. Nach erfolgter Reposition in Analgosedierung oder Kurznarkose ist nach abermaliger Überprüfung des Gefäßstatus die Stabilitätskontrolle unter Durchleuchtung obligat. Bei höhergradiger Instabilität kann es bei fortschreitender Extension $\mathrm{zu}$ einer Reluxation des Ellenbogengelenkes kommen. Valgus- und Varusstabilität sollten standardisiert unter Durchleuchtung in voller Streckung und in $20^{\circ}$-Beugung in Pro- und Supination getestet werden ([27]; - Abb. 3). Anschließend erfolgt die Ruhigstellung im Oberarmgips. Zur Dokumentation und Stellungskontrolle sowie zur Beurteilung der knöchernen Strukturen wird der Ellenbogen anschließend nochmals in 2 Ebenen geröntgt. 
Zur Beurteilung des Kapsel-BandApparates sollte als weiterführendes Diagnostikum eine Magnetresonanztomographie (MRT) durchgeführt werden [8]. Begleitverletzungen wie beispielsweise chondrale Läsionen können im MRT ebenfalls gut beurteilt werden. Die Aussagekraft der MRT hinsichtlich der Kollateralbänder ist jedoch durch Faktoren wie die Stellung des Gelenkes (schmerzbedingt meist $90^{\circ}$-Flexionsstellung) beeinträchtigt [6]. Hinzu kommt, dass wichtige ligamentäre Strukturen wie das LUCL aufgrund des schrägen Verlaufes im MRT nur unzuverlässig dargestellt werden können [30].

In der konventionellen Röntgendiagnostik und im MRT sollte auf sog. Warnzeichen für eine Instabilität geachtet werden. Ein dorsaler Versatz des Radiuskopfes zum Capitulum humeri ist ein Hinweis auf eine PLRI (- Abb. 4a; [5]). Eine Inkongruenz des Olekranons in der Trochlea stellt ein weiteres wichtiges Instabilitätsmerkmal im MRT dar (• Abb. 4b; [27]). Ein weiterer Hinweis für eine Ruptur des LUCL mit resultierender Instabilität ist das sog. „drop sign“, womit die Erweiterung des Gelenkspaltes zwischen Ulna und Trochlea auf über $4 \mathrm{~mm}$ bezeichnet wird (-Abb. 4c; [1]).

In der Fachliteratur besteht kein Konsens bezüglich der optimalen Therapie der akuten, ligamentären Ellenbogeninstabilität [4]. Die Bandbreite der Therapieoptionen reicht von der Ruhigstellung im Gips bis hin zur primären Bandrekonstruktion und Anlage eines Fixateur externe.

Als Therapiestandard in der Behandlung der einfachen Ellenbogenluxation ist die kurzzeitige Ruhigstellung des Gelenks mit anschließender funktioneller Nachbehandlung derzeit am weitesten verbreitet. Eine konservative Therapie bei einfachen Ellenbogenluxationen ist nach unserer Erfahrung dann sinnvoll, wenn im funktionellen Bogen zwischen $130^{\circ}$ und $30^{\circ}$-Beugung keine Tendenz zur Reluxation besteht und in der Stabilitätsprüfung unter Durchleuchtung keine ausgeprägte Varus- oder Valgusinstabilität nachzuweisen ist. In einer eigenen Studie konnten wir zeigen, dass bei einer medialen oder lateralen Aufklappbarkeit von unter $10^{\circ}$ in der initialen Durch-

Trauma Berufskrankh 2016 · [Suppl 4]: 18:S332-S339 DOI 10.1007/s10039-016-0145-6

c) Springer-Verlag Berlin Heidelberg 2016

\section{Schnetzke · T. Guehring · P. A. Grützner}

\section{Diagnostik und Therapie der akuten und chronischen Ellenbogeninstabilität}

\section{Zusammenfassung}

Das Ellenbogengelenk ist eine komplexe Verbindung drei verschiedener Gelenke. Die Kongruenz und Stabilität hängen von der Integrität der knöchernen Artikulationspartner sowie von ligamentären, kapsulären und muskulären Strukturen ab. Instabilitäten des Ellenbogens resultierten am häufigsten infolge akuter Verletzungen der knöchernen und weichteiligen stabilisierenden Strukturen. Rezidivierende Mikrotraumata oder Überlastungen z. B. bei Wurfsportarten können zu chronischen Instabilitäten mit hieraus resultierenden Beschwerden am
Ellenbogengelenk führen. Das primäre Ziel bei der Behandlung von Instabilitäten des Ellenbogengelenkes ist die Wiederherstellung eines kongruenten und stabilen Gelenkes. Im vorliegenden Beitrag werden die wichtigsten diagnostischen und therapeutischen Schritte bei akuten und chronischen Instabilitäten des Ellenbogens dargestellt.

Schlüsselwörter

Ellenbogengelenk · Verletzungen · Nachbehandlung $\cdot$ Mikrotraumata $\cdot$ Biomechanik

\section{Diagnostics and therapy of acute and chronic elbow instability}

\section{Abstract}

The elbow joint is a complex connection of three different joints. Congruity and stability of the elbow joint depend on the integrity of the articular bony structures, the collateral ligaments and the extensor and flexor tendons of the forearm. In most cases instabilities of the elbow joint result from damage to the stabilizing bony and ligamentous structures. Recurrent microtrauma and overuse, e.g. in throwing sports, can lead to chronic instability resulting in complaints of the elbow joint. The primary goal in the treatment of elbow instabilities are the restoration of a stable and congruent joint. The aim of this review is to present the main diagnostic and therapeutic steps in the treatment of acute and chronic instabilities of the elbow joint.

Keywords

Elbow joint · Injuries - Aftercare $\cdot$ Biomechanics $\cdot$ Microtraumata leuchtung mit einem exzellenten klinischen Ergebnis nach konservativer Therapie gerechnet werden kann [27].

Spätestens nach einer Woche sollte eine klinische und bildgebende Kontrolluntersuchung bezüglich der Stabilität und Beweglichkeit des Ellenbogengelenkes erfolgen. Erscheint die Artikulation im Ellenbogengelenk regelrecht und besteht bereits ein gutes aktives Bewegungsausmaß, kann eine Ellenbogengelenkorthese angelegt werden. Hieraus erfolgt dann die aktive Beübung ohne Bewegungseinschränkung unter physiotherapeutischer Anleitung für insgesamt 6 Wochen. Eine Ruhigstellung über 2 Wochen hinaus sollte zur Vermeidung einer posttraumatischen Arthrofibrose unbedingt vermieden werden [12].

Die Indikation zur primären Bandrekonstruktion besteht nach gängiger
Literatur bei fehlender Gelenkkongruenz nach geschlossener Reposition oder verbleibender höhergradiger Instabilität nach Reposition mit Reluxation bei mittlerer Streckung (30-45 $)$ [15].

Der Terminus der Reluxationstendenz bzw. der verbleibenden höhergradigen Instabilität ist dabei nicht genau definiert und führt daher zu einem uneinheitlichen Vorgehen [4]. Nach unserer eigenen Erfahrung und nach Auswertung unserer eigenen Ergebnisse besteht bereits bei einer lateralen oder medialen Aufklappbarkeit des Gelenkes von mehr als $10^{\circ}$ in der initialen Durchleuchtung eine höhergradige Instabilität des Gelenkes. Eine konservative Therapie ist in diesem Fall mit einer deutlich erhöhten Komplikationsrate hinsichtlich chronischer Instabilitäten und konsekutiver Arthrofibrose vergesellschaftet [27]. Wir empfehlen da- 

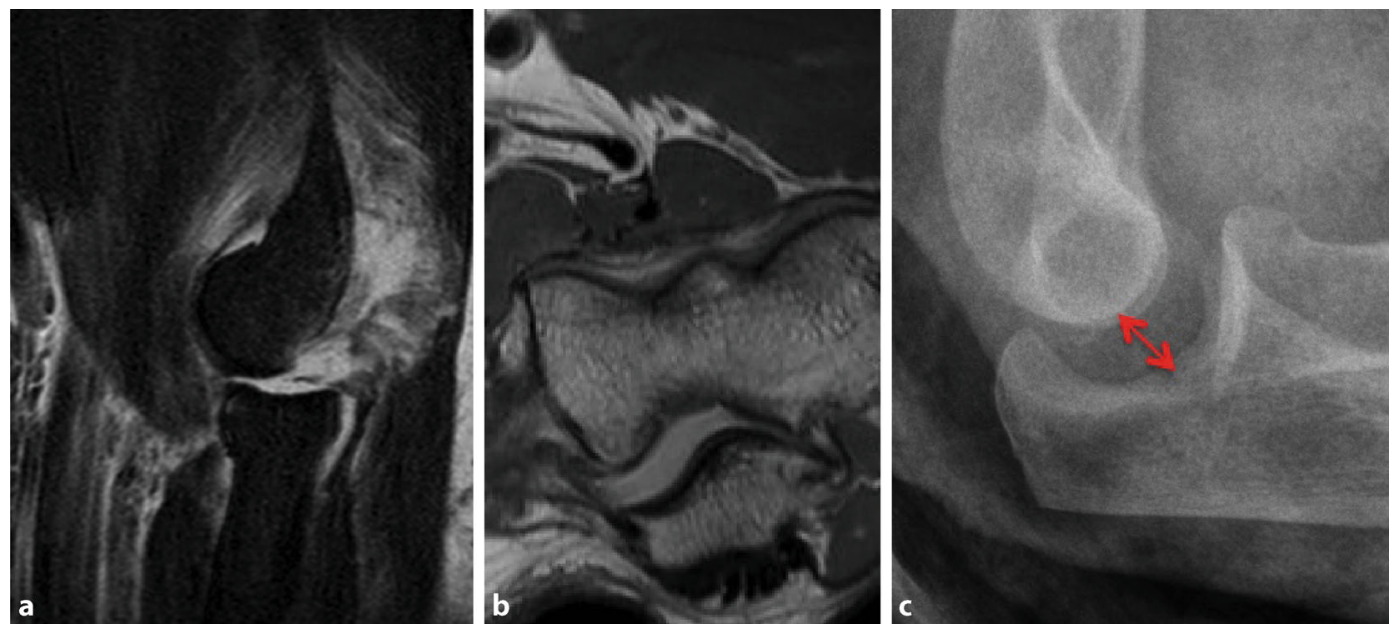

Abb. $4 \varangle$ Warnzeichen für Instabilität: a Subluxation des Radiuskopfes nach dorsal, b ulnohumerale Inkongruenz, c Erweiterung des ulnohumeralen Gelenkspaltes (roter Pfeil), sog. "drop sign"

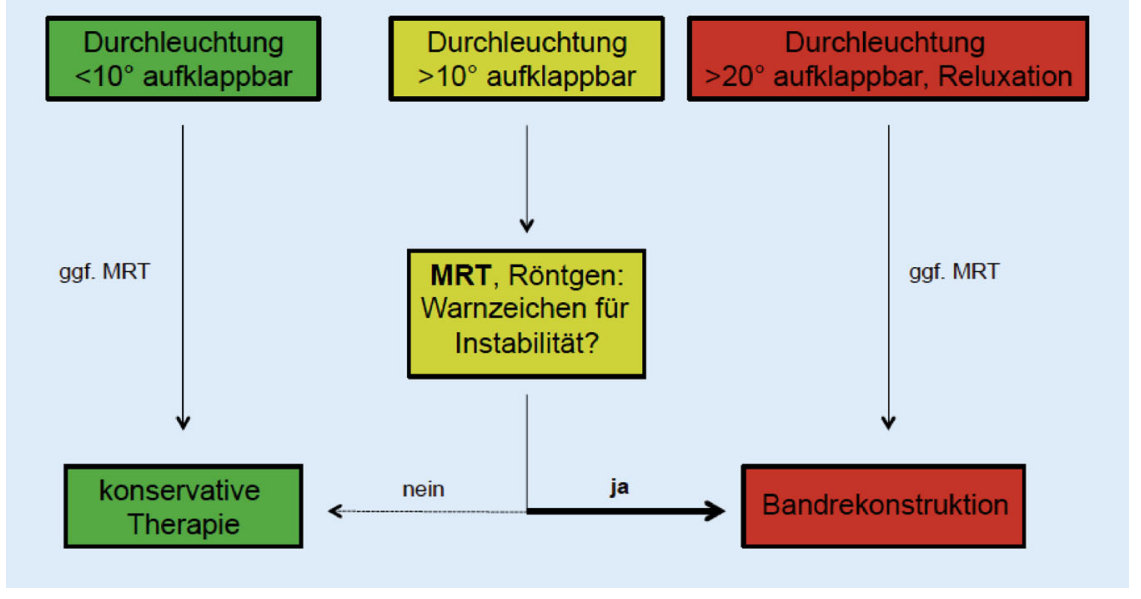

Abb. 5 ॥ Therapiealgorithmus bei der akuten, einfachen Instabilität. MRT Magnetresonanztomographie

her bei einer medialen oder lateralen Aufklappbarkeit von mehr als $10^{\circ}$ unter der Durchleuchtung eine primäre Bandrekonstruktion zur Vermeidung posttraumatischer Komplikationen. Unser Vorgehen bei ligamentären Instabilitäten ist anhand eines Algorithmus in - Abb. 5 veranschaulicht.

Individuelle Faktoren wie das Patientenalter und die körperlichen Anforderungen im Alltag und im Sport sollten bei der Therapiefindung zusätzlich berücksichtigt werden. Abhängig von den verletzten Strukturen wird der operative Zugang von lateral oder medial gewählt. Am häufigsten ist der LCL-Komplex betroffen, weshalb für den Zugang von den meisten Autoren das sog. Kocher-Intervall - zwischen M. extensor carpi ulnaris und M. anconaeus - gewählt wird. Die bei medialen oder lateralen Bandruptu- ren oft auch mit betroffenen Extensorenund Flexorensehnen sollten unbedingt rekonstruiert und mit Ankersystemen refixiert werden, da sie eine wichtige Rolle für die Ellenbogenstabilität spielen [17]. Besteht nach der Bandnaht und der Kapselrefixation eine persistierende Instabilität, sollte additiv ein Bewegungsfixateur angebracht werden [10].

\section{Akute, knöcherne Instabilität}

Das Vorgehen bei der akuten, knöchernen Instabilität, sog. komplexe Ellenbogeninstabilität, entspricht zunächst demjenigen bei der einfachen Luxation. Finden sich im konventionellen Röntgenbild Hinweise auf intraartikuläre knöcherne Begleitverletzungen, besteht die Indikation zur Durchführung einer Computertomographie (CT). Die CT erlaubt ein besseres Frakturverständnis und damit eine bessere Therapie- und Operationsplanung. Eine Stabilitätsprüfung unter Durchleuchtung sollte in diesem Fall unterlassen werden.

Grundsätzlich gilt bei den komplexen Instabilitäten, dass zur Stabilisierung des Ellenbogens die knöcherne Integrität des ulnohumeralen Gelenkes wiederhergestellt werden muss [31]. Das Vorgehen ist dabei abhängig vom Ausmaß der knöchernen Verletzung. Im folgenden Abschnitt wird das Vorgehen bei - Beteiligung des Radiuskopfes,

- Beteiligung des Proc. coronoideus,

- Beteiligung des Olekranons und - der sog. Terrible-triad-Verletzung

dargestellt.

\section{Komplexe Instabilitäten mit Radiuskopffraktur}

Der Radiuskopf ist ein wichtiger sekundärer Stabilisator des Ellenbogengelenkes gegen Valgusstress. Im Falle einer komplexen Instabilität mit gleichzeitiger Ruptur des medialen Kollateralbandes übernimmt der Radiuskopf die Funktion eines primären Stabilisators [14]. Die Rekonstruktion des Radiuskopfes im Zusammenhang mit einer Luxation ist daher zur Wiederherstellung der Stabilität des Ellenbogens von zentraler Bedeutung. Primär sollte eine Rekonstruktion des Radiuskopfes mittels Schrauben- oder Plattenosteosynthese angestrebt werden. Gelingt jedoch keine anatomische Reposition, sollte frühzeitig ein Ersatz durch eine Radiuskopfprothese in Erwägung gezogen werden [24]. Unsere eigenen Ergeb- 


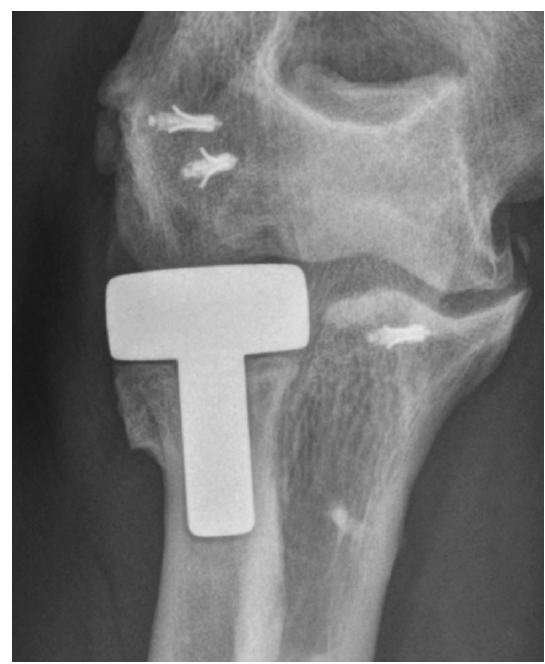

Abb. $6 \Delta$ Oversizing der Radiuskopfprothese mit ulnohumeraler Inkongruenz

nisse zeigen, dass eine korrekt implantierte Radiuskopfprothese vergleichbare Ergebnisse wie eine anatomische Rekonstruktion des Radiuskopfes erzielen kann [26]. Kritisch ist der Einsatz der Radiuskopfprothese bei Patienten mit handwerklichen Berufen, da nach prothetischem Radiuskopfersatz je nach Hersteller eine Belastungsgrenze von ca. $7 \mathrm{~kg}$ vorgeschrieben ist. Bei der Implantation einer Radiuskopfprothese sollte ein sog. Oversizing unbedingt vermieden werden, da dies zu einem erhöhten Druck auf das Capitulum humeri mit konsekutiven Schmerzen und Bewegungseinschränkung führt (• Abb. 6; [26]).

Folgendes Vorgehen wird bei komplexer Instabilität mit Radiuskopffraktur empfohlen:

1. Rekonstruktion des Radiuskopfes, wenn eine anatomische Rekonstruktion möglich ist,

2. bei Nicht-Rekonstruierbarkeit Implantation einer Radiuskopfprothese,

3. Rekonstruktion des LCL-Komplexes,

4. bei persistierender medialer Instabilität: Rekonstruktion des MCL,

5. bei persistierender Instabilität: Bewegungsfixateur.

\section{Komplexe Instabilitäten mit Fraktur des Processus coronoideus}

Der Proc. coronoideus ist ein wichtiger primärer Stabilisator des Ellenbogengelenkes und sollte daher bei Vorliegen einer komplexen Instabilität rekonstruiert

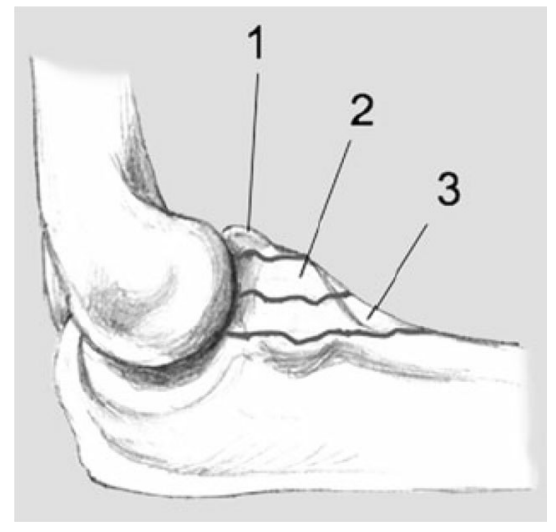

Abb. $7 \Delta$ Klassifikation nach Regan und Morrey: Typ 1 Avulsion der Spitze, Typ 2 weniger als 50\% des Koronoids betreffend, Typ 3 basisnah, mehr als $50 \%$ des Koronoids betreffend. (Aus [22])

werden [31]. Die Klassifikation nach Regan und Morrey ist im klinischen Alltag am gebräuchlichsten (• Abb. 7).

Generell gilt, dass eine Proc.-coronoideus-Fraktur Typ II und III immer refixiert werden sollte. Hinsichtlich der optimalen Therapie bei Avulsionsfrakturen vom Typ I besteht nach Literaturangaben Uneinigkeit [21]. Zur Refixierung werden verschiedene Techniken beschrieben, wobei bei größeren Fragmenten die indirekte Verschraubung von dorsal aufgrund der geringen Zugangsmorbidität Vorteile gegenüber der direkten Verschraubung hat [13]. Den Nachteil der schlechteren Visualisierung der Reposition kann man evtl. durch eine intraoperative 3D-Bildgebung kompensieren.

O'Driscoll hat eine weitere Klassifikation der Koronoidfrakturen beschrieben, die auch die Verletzungsmechanismen und die Ansätze der Kapsel-BandStrukturen (Ansatz des MCL an der anteromedialen Facette des Koronoids) mit einbezieht [23].

Das empfohlene Vorgehen bei komplexer Instabilität mit Koronoidfraktur ist:

- Typ-I-Frakturen nach Regan und Morrey:

- Zugang wählen je nach vorherrschender Bandinstabilität,

- bei Typ-I-Frakturen Refixation des Proc. coronoideus mit einem Fadenanker,
- Rekonstruktion des lateralen/ medialen Bandapparates je nach Instabilität;

- Typ-II/III-Frakturen:

- bei isolierter Koronoidfraktur indirekte Verschraubung von dorsal,

- bei begleitender medialer Instabilität ulnarer Zugang mit Darstellung des N. ulnaris, direkte Schraubenoder Plattenosteosynthese der Koronoidfraktur, Rekonstruktion des MCL,

- bei Nicht-Rekonstruierbarkeit Verwenden des Radiuskopfes oder eines Beckenkammblockes als Neo-Koronoid.

\section{Komplexe Instabilitäten mit Fraktur des Olekranons}

Transolekranonluxationsfrakturen entstehen häufig infolge von Hochrasanztraumata. Grundsätzlich kann bei dieser Form der komplexen Instabilität alleine durch die Osteosynthese des Olekranons eine ausreichende Stabilität des Ellenbogengelenkes wiederhergestellt werden [31]. Wichtig ist es, hierbei auch auf eine Beteiligung des Koronoids zu achten und in diesem Fall das Koronoid durch die Osteosynthese mit zu stabilisieren.

Das empfohlene Vorgehen bei komplexer Instabilität mit Olekranonfraktur ist:

- stabile, anatomische Rekonstruktion des Olekranons,

- bei Beteiligung des Koronoids: indirekte Schraubenosteosynthese des Koronoids,

- Lagerung in einer Oberarmschiene bis zur Wundheilung,

- frühfunktionelle Mobilisierung.

\section{Komplexe Instabilitäten mit sog Terrible-triad-Verletzung}

Komplexe Instabilitäten mit Frakturen des Radiuskopfes und des Koronoids und Ruptur des medialen und/oder lateralen Kollateralbandes werden als sog. Terrible-triad-Verletzung bezeichnet [31]. Die Bezeichnung ist historisch und begründet sich auf die schlechten funktionellen Ergebnisse, die früher mit dieser Verletzungskombination einhergingen. In den häufigsten Fällen liegt dies an ei- 

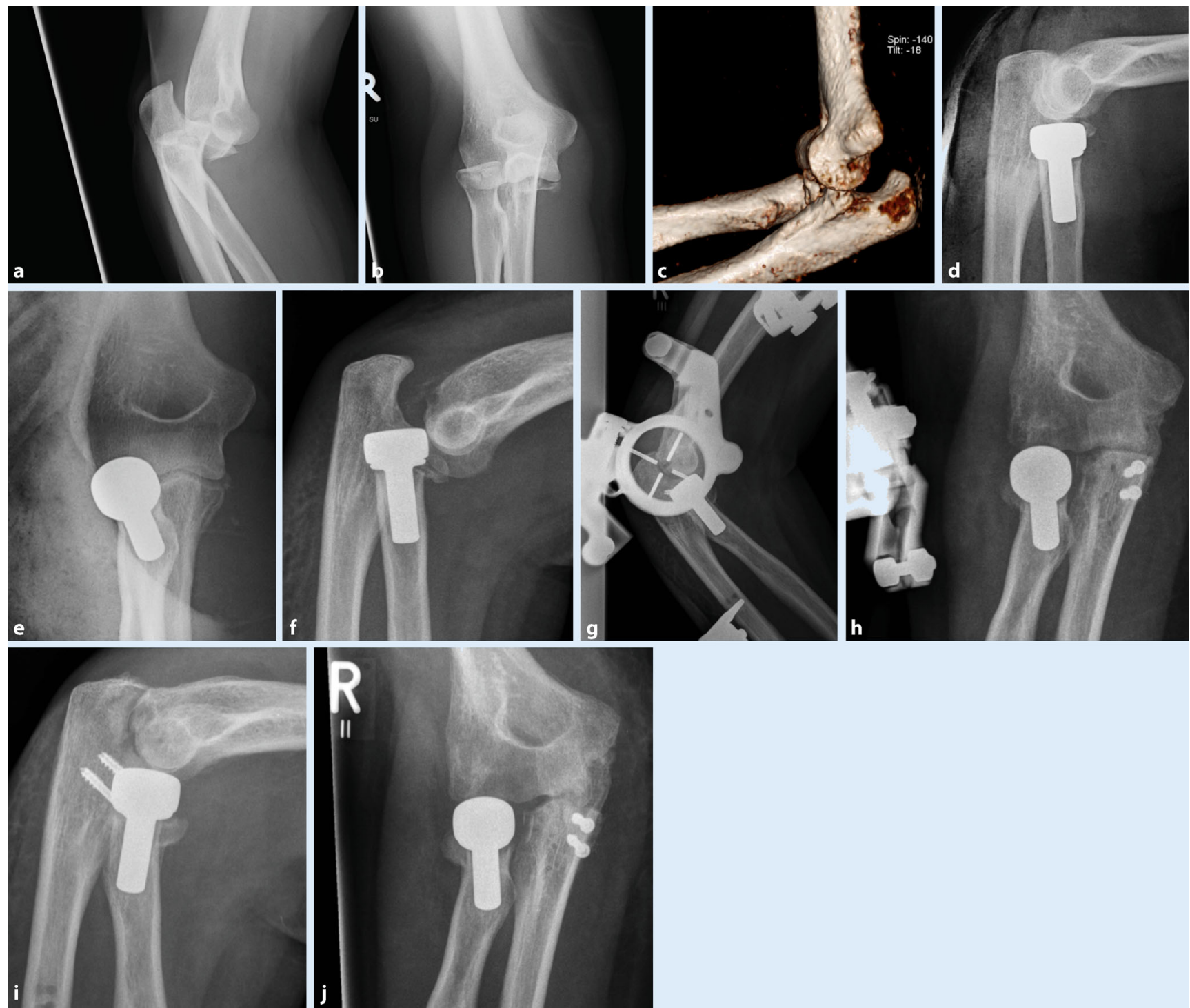

Abb. $8 \Delta$ a-c Terrible-triad-Verletzung mit Radiuskopffraktur, Proc.-coronoideus-Fraktur Typ II nach Regan und Morrey. d, e Auswärtige Versorgung mit Radiuskopfprothese. f Röntgenkontrolle eine Woche postoperativ zeigt eine persistierende Instabilität. g, h Patientin wird operativ revidiert und erhält eine Koronoidplastik aus einem autologen Beckenkammblock, additiv Anlage eines Bewegungsfixateurs für 6 Wochen. i, j Nach Abnahme des Fixateurs ist das Gelenk stabil und kongruent

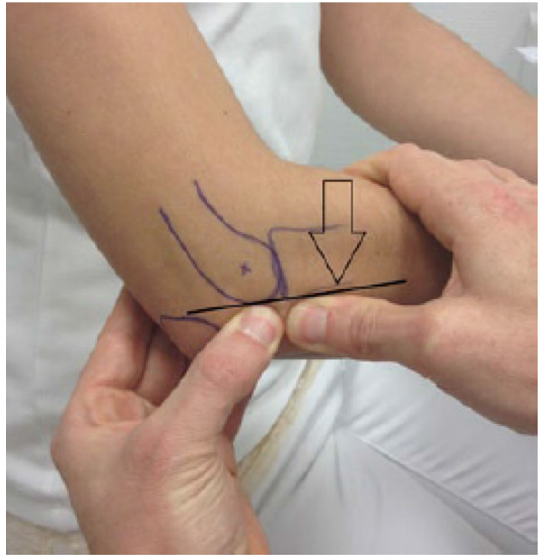

Abb. 9 ॥ Pinzettengriff. (Aus [9]) ner unzureichenden Stabilisierung des Proc. coronoideus. Ab einer Beteiligung von mehr als $50 \%$ der Koronoidhöhe ist eine ausreichende Stabilisierung des Ellenbogens ohne Adressierung des Koronoids nicht möglich (Fallbeispiel in - Abb. 8a-e; [14]).

Mithilfe eines standardisierten Vorgehens bei der Behandlung der Terribletriad-Verletzung können deutlich bessere Ergebnisse erzielt werden. Das Ziel der operativen Therapie ist zunächst die knöcherne Rekonstruktion des Ellenbogens. Damit wird die „komplexe in eine einfache“ Instabilität überführt. Der la- terale Kollateralbandkomplex sollte anschließend immer rekonstruiert werden. Hinsichtlich des MCL herrscht dagegen Uneinigkeit [31]. Nach unserer Erfahrung sollte das MCL im Falle einer Ruptur ebenfalls rekonstruiert werden, um chronische Instabilitäten zu vermeiden.

Das empfohlene Vorgehen bei komplexer Instabilität mit Terrible-triad-Verletzung ist:

- knöcherne Rekonstruktion des Radiuskopfes und des Proc. coronoideus,

- Rekonstruktion des LCL-Komplexes,

- Rekonstruktion des MCL bei persistierender Instabilität, 

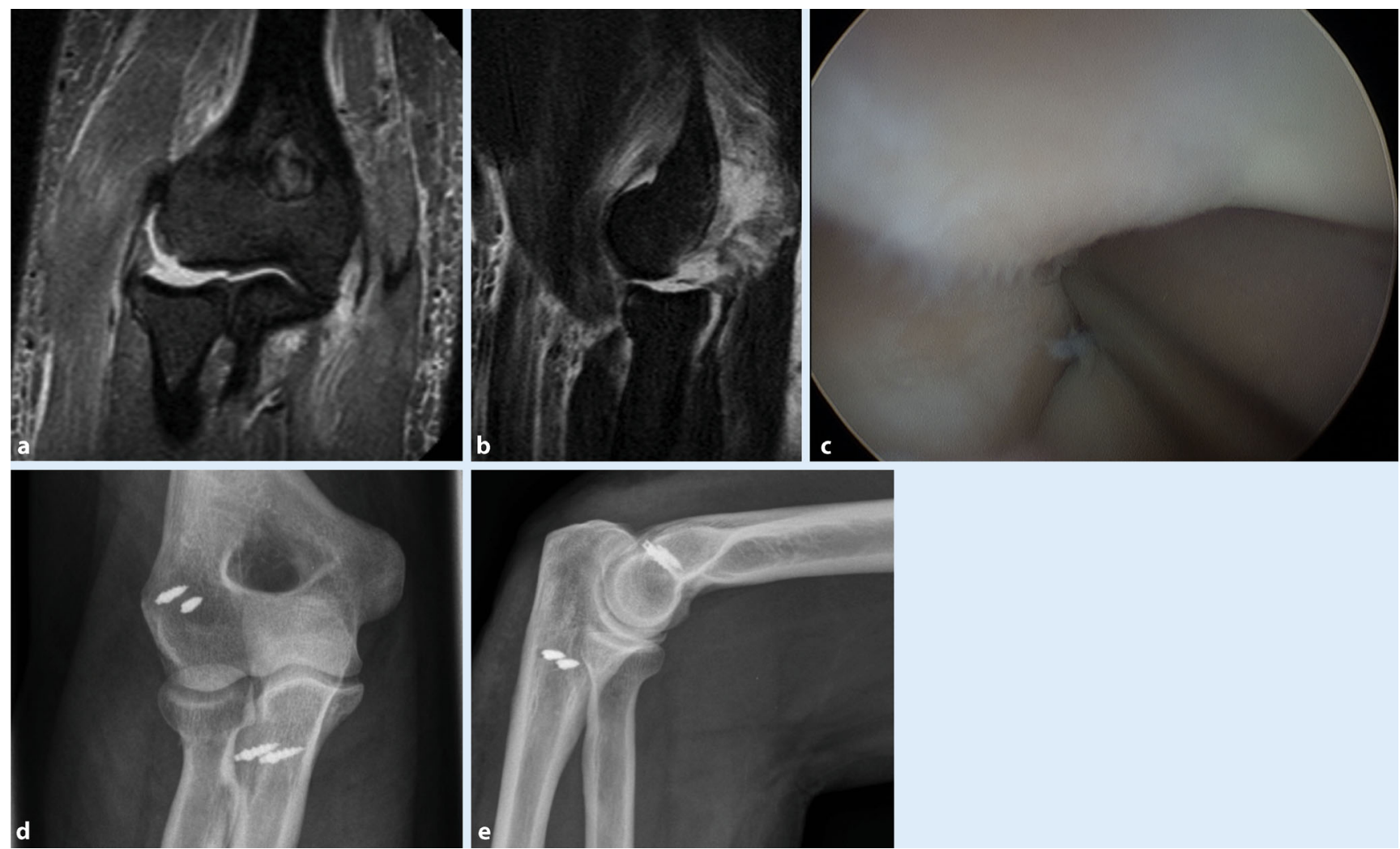

Abb. $10 \Delta$ a, b Patient mit persistierenden Beschwerden nach konservativer Therapie einer einfachen Ellenbogenluxation. In der Magnetresonanztomographie 8 Wochen nach Trauma besteht eine radiohumerale Inkongruenz als Hinweis auf eine PLRI. c In der Arthroskopie bestätigt sich die Diagnose der PLRI mittels Wechselstabtest. d, e Postoperative Kontrolle nach Durchführung einer LUCL-Rekonstruktion mit Trizepsstreifen: kongruentes Gelenk

— additiver Bewegungsfixateur bei persistierender Instabilität.

\section{Chronische Instabilität}

Bei der chronischen Instabilität haben die Anamnese und klinische Untersuchung einen sehr hohen Stellenwert [9]. Anamnestisch berichten die Patienten häufig von stattgehabten Traumata oder stellen eine Risikogruppe (z. B. Wurfsportler) für chronische Instabilitäten dar. Die klinische Untersuchung der Ellenbogeninstabilität ist am wachen Patienten durch die muskuläre Vorspannung zwar erschwert, gibt jedoch erste Hinweise auf eine mögliche Instabilität. Die Stabilitätsprüfung sollte wie auch bei der akuten, einfachen Luxation in Streckung und in einer leichten Beugung von etwa $20-30^{\circ}$ erfolgen [9]. Ein sensibler Test für die PLRI ist der sog. "Pinzettengriff“ (• Abb. 9). Dieses Manöver entspricht der posterolateralen Rotationsbewegung, die sich bei einer entsprechenden Instabilität provozieren lässt. Anhand der relativen Bewegung zwischen den beiden Daumen lässt sich die Ausprägung der Instabilität abschätzen.

In der konventionellen Röntgendiagnostik und im MRT lassen sich bei Vorliegen einer PLRI bereits Hinweise für eine chronische Ellenbogeninstabilität finden (•Tab. 2). Die Arthroskopie ist das sensibelste diagnostische Verfahren, mit dem auch geringste Instabilitätsformen, die sich nur unter dynamischer Testung mit dem Wechselstab darstellen lassen, nachgewiesen werden können (Fallbeispiel in - Abb. 10a-e). Im Zweifel oder bei unklaren Fällen sollte vor stabilisierenden Eingriffen stets eine Arthroskopie zur Erhebung der Gelenkinstabilität und des Gelenkstatus durchgeführt werden. In der Arthroskopie zeigen sich bereits eingetretene Knorpelschäden, die bei chronischen Instabilitäten bzw. nach zurückliegenden Luxationsverletzungen vorliegen können. Solche Gelenkschäden können einen direkten Einfluss auf das Behandlungskonzept und die zu erwartende Prognose für das Gelenk haben. Bei bereits zu starker Gelenkschädigung ist ein stabilisierender Eingriff nicht mehr sinnvoll.

Die Bandplastik ist eine etablierte Therapieoption bei chronischer Instabilität im Ellenbogengelenk [2]. Bei der lateralen sowie der medialen Ersatzplastik stehen generell die Palmaris-longus-Sehne oder die Gracilissehne zur Verfügung. Einige Autoren bevorzugen die Verwendung eines Trizepsstreifens zur Rekonstruktion des LUCL, womit gute bis sehr gute Ergebnisse erzielt werden können [2].

\section{Fazit für die Praxis}

\section{- Das primäre Ziel der Behandlung von Ellenbogeninstabilitäten ist die Wiederherstellung eines stabilen und kongruenten Gelenkes, das eine}


schnellstmögliche frühfunktionelle Nachbehandlung ermöglicht.

- Zur erfolgreichen Therapie der Ellenbogeninstabilität sind ein Verständnis für die biomechanischen Zusammenhänge und eine gezielte präoperative Diagnostik unerlässlich.

\section{Korrespondenzadresse}

\section{Prof. Dr. med. P. A. Grützner}

BG - Klinik Ludwigshafen

Ludwig-Guttmann-Str. 13, 67071 Ludwigshafen, Deutschland

paul.gruetzner@bgu-ludwigshafen.de

\section{Einhaltung ethischer Richtlinien}

Interessenkonflikt. M. Schnetzke, T. Guehring und P.A. Grützner geben an, dass kein Interessenkonflikt besteht.

Dieser Beitrag beinhaltet keine von den Autoren durchgeführten Studien an Menschen oder Tieren.

The supplement containing this article is not sponsored by industry.

\section{Literatur}

1. Coonrad RW, Roush TF, Major NM et al (2005) The drop sign, a radiographic warning sign of elbow instability. J Shoulder Elbow Surg 14:312-317

2. Dehlinger Fl, Ries C, Hollinger B (2014) LUCL reconstruction using a triceps tendon graft to treat posterolateral rotatory instability of the elbow. Oper Orthop Traumatol 26:414-427

3. Duckworth AD, Ring D, Kulijdian A et al (2008) Unstable elbow dislocations. J Shoulder Elbow Surg 17:281-286

4. Hackl M, Beyer F, Wegmann K et al (2015) The treatment of simple elbow dislocation in adults - a systematic review and meta-analysis. Dtsch Arztebl Int 112:311-319

5. Hackl M, Wegmann K, Ries C et al (2015) Reliability of magnetic resonance imaging signs of Posterolateral rotatory instability of the elbow. J Hand Surg Am 40:1428-1433

6. Hauptfleisch J, English C, Murphy D (2015) Elbow magnetic resonance imaging: imaging anatomy and evaluation. Top Magn Reson Imaging 24:93-107

7. Hildebrand KA, Patterson SD, King GJ (1999) Acute elbow dislocations: simple and complex. Orthop Clin North Am 30:63-79

8. Ho CP (1995) Sports and occupational injuries of the elbow: MR imaging findings. AJR Am J Roentgenol 164:1465-1471

9. Hollinger B, DehlingerF, FrankeS (2014) Diagnostik und Therapie der ligamentären Ellenbogeninstabilitäten. Obere Extremität 9:147-155

10. Hopf JC, Berger V, Krieglstein CF et al (2015) Treatment of unstable elbow dislocations with hinged elbow fixation-subjective and objective results. J Shoulder Elbow Surg 24:250-257
11. Kuhn MA, Ross $G$ (2008) Acute elbow dislocations. Orthop Clin North Am 39:155-161

12. Mehlhoff TL, Noble PC, Bennett JB et al (1988) Simple dislocation of the elbow in the adult. Results after closed treatment. J Bone Joint Surg Am 70:244-249

13. Moon JG, Zobitz ME, An KN et al (2009) Optimal screw orientation for fixation of coronoid fractures. J Orthop Trauma 23:277-280

14. Morrey BF (2009) Current concepts in the management of complex elbow trauma. Surgeon 7:151-161

15. Morrey BF (2012) Ligament injury and the use of hinged external fixators at the elbow. Instr Course Lect 61:215-225

16. O'Driscoll SW (2000) Classification and evaluation of recurrent instability of the elbow. Clin Orthop Relat Res 370:34-43

17. O'Driscoll SW (1999) Elbow instability. Acta Orthop Belg 65:404-415

18. O'Driscoll SW (1994) Elbow instability. Hand Clin 10:405-415

19. O'Driscoll SW, Bell DF, Morrey BF (1991) Posterolateral rotatory instability of the elbow. J Bone Joint Surg Am 73:440-446

20. O'Driscoll SW, Jupiter JB, King GJ et al (2001) The unstable elbow. Instr Course Lect 50:89-102

21. Papatheodorou LK, Rubright JH, Heim KA et al (2014) Terrible triad injuries of the elbow: does the coronoid always need to be fixed? Clin Orthop Relat Res 472:2084-2091

22. Partenheimer A, Geisler A, Voigt C et al (2007) Luxation, instabilityand dislocation fractures of the elbow joint. Trauma Berufskrankh 9:S197-S201

23. Pollock JW, Brownhill J, Ferreira L et al (2009) The effect of anteromedial facet fractures of the coronoid and lateral collateral ligament injury on elbow stability and kinematics. J Bone Joint Surg Am 91:1448-1458

24. Ring D, Quintero J, Jupiter JB (2002) Open reduction and internal fixation of fractures of the radial head. J Bone Joint Surg Am 84:1811-1815

25. Saltzman BM, Chalmers PN, Mascarenhas R et al (2014) Upper extremity physeal injury in young baseball pitchers. Phys Sportsmed 42:100-111

26. Schnetzke M, Aytac S, Deuss M et al (2014) Radial head prosthesis in complex elbow dislocations: effect of oversizing and comparison with ORIF. Int Orthop 38:2295-2301

27. Schnetzke M, Aytac $S$, Studier-Fischer $S$ et al (2015) Initial joint stability affects the outcome after conservative treatment of simple elbow dislocations: a retrospective study. J Orthop Surg Res 10:128

28. Schreiber JJ, Warren RF, Hotchkiss RN et al (2013) An online video investigation into the mechanism of elbow dislocation.J Hand Surg Am 38:488-494

29. Siebenlist S, Biberthaler P (2013) Acute soft tissue injuries of the elbow. Trauma Berufskrankh 17:132-139

30. Singer AD, Hanna T, Jose J et al (2015) A systematic, multimodality approach to emergency elbow imaging. Clin Imaging 40(1):13

31. Tarassoli P, Mccann P, Amirfeyz R (2013) Complex instability of the elbow. Injury. doi:10.1016/j.injury.2013.09.032 